\title{
Some Aspects of Analysis of Dolphins' Acoustical Signals
}

\author{
Vyacheslav Ryabov \\ Karadag Natural Reserve of NAS of Ukraine, Kurortnoe, Feodosia, Ukraine \\ E-mail: ryaboff@ukr.net \\ Received August 3, 2011; revised August 19, 2011; accepted August 22, 2011
}

\begin{abstract}
Dolphins produce various types of sounds in a wide range of frequencies. Characteristics of some sounds till now have not been correctly registered, that influenced on interpretation of their functions. Studying of the characteristics and functions of dolphins' acoustical signals is the purpose of the present work. In this work the acoustical signals of two dolphins (Tursiops truncatus) were registered by two-channel system in the frequencies band up to $200 \mathrm{kHz}$ at quasi-stationary position of the dolphins. The dolphins along with whistles are producing the packs of coherent and non-coherent broadband pulses. The waveform and spectrum of coherent pulses was invariable within a pack, but considerably varies from a pack to a pack. The waveform of each non-coherent pulse vary from a pulse to a pulse in each pack, therefore their spectrum also vary from a pulse to a pulse and have many extremums in the band of $6-200 \mathrm{kHz}$. It is very likely that the non-coherent pulses play a part of phonemes of a dolphin spoken language and the probing signals of dolphin's noncoherent sonar. The use possibility of the signals by dolphins for communication and orientation was considered, as the signals apparently are bimodal. Results of the work have significance for further studying of the dolphin's sonar and spoken language.
\end{abstract}

Keywords: Dolphins, Hearing, Sonar, Acoustical Signals, Language, Orientation

\section{Introduction}

Cetaceans are secondary-aquatic mammals which have completely returned to living in the sea. It is supposed, that they evolved from primitive ungulates, or it is probable, from carnivores which foraged in coastal waters until completely adapting to a complete life in the sea, approximately 50 - 70 million years ago [1-4]. The fossil records of the early Oligocene shows evidence of divergence between the suborders Odontoceti and Mysticeti. Till now there are at least 65 recognized species of Odontoceti which represent carnivores $[5,6]$.

It is known that Odontoceti undergo a number of functional and morphological modifications in the process of secondary adaptation to the aquatic habitat. It is tempting to think that the most vivid modification known as telescoping of a skull [7] was associated, among other reasons, with the development of the own echo-location system and peripheral part of hearing, while the convergence of the lower-jaw halves at the level of mental foramens reflects the optimization of aperture and base of the new external ear [8-11].

In Cetaceans the encephalization hypothetically arise rapidly and already 25 million years ago they had a brain weight more than of Homo sapiens. Average weight of a dolphin brain of $1587 \mathrm{~g}$, it is more convoluted and having more surface area per unit of volume than a human brain [12].

Though experiments with dolphins are conducting from the middle of the last century, questions about necessity of such brain as well as about existence possibility of a highly-developed language and supreme forms of a consciousness are still discussing.

In the same time there were achieved certain successes in the studying of dolphins' acoustical signals. The tonal frequency-modulated whistles and packets of short broadband acoustical pulses or the pulse burst are thought to be the sounds primarily playing a role in social interaction of dolphins [13-21]. However, these acoustical signals mostly were studied in a frequencies band up to $20 \mathrm{kHz}$, therefore, the authors practically in all these works, discussed them as signals with the majority of energy on frequencies from few hundred to a few thousand Hz. Recently some of these acoustical signals are studied in more details in frequencies band up to $130 \mathrm{kHz}[22,23]$. Authors have shown, that free-ranging the Hawaiian spinner dolphin (Stenella longirostris) and the Atlantic spotted dolphin (Stenella frontalis) are pro- 
ducing the packs of short broadband pulses (the burst pulses), on the average approximately 30 and 100 pulses, with a mean interpulse interval $3.85 \mathrm{~ms}$ and $3.19 \mathrm{~ms}$, respectively. The spectrum maximum and the central frequency of the pulses for spinner dolphin were on average 32.3 and $40.1 \mathrm{kHz}$, respectively, for spotted dolphins these were somewhat higher at 40.3 and $44.4 \mathrm{kHz}$, respectively. The power spectrum width of these pulses roughly $20.5 \mathrm{kHz}$ for spinner dolphin and $18.1 \mathrm{kHz}$ for spotted dolphins. Spectral energy distribution in the pulses did not depend on position of the pulse in a pack or an interpulse interval. Only less than $20 \%$ of pulses had peak of energy on frequencies below $20 \mathrm{kHz}$. On the average more than $80 \%$ of pulses energy was on frequencies above $20 \mathrm{kHz}$ for both species. At the same time authors have shown, that the waveforms of these pulses and the dolphin's sonar clicks could not be readily distinguished from the other. Therefore, click train were considered as burst pulses if their mean interpulse interval did not exceed $10 \mathrm{msec}$. Dolphins produced these pulses primarily on close distances to each other up to $3-14 \mathrm{~m}$, therefore authors have assumed, that dolphins use these pulses for exchange of emotional signals [23].

Thus, the dolphin's acoustical signals have been registered mainly incorrectly (inadequate band of frequencies and levels of records that lead to clipping and distortion of a signal's waveform; random position of a dolphin relatively of a hydrophon). Therefore in this work the acoustical signals of two dolphins (Tursiops truncatus) were registered by the two-channel system with a wide dynamic range in the frequencies band up to $200 \mathrm{kHz}$ at quasi-stationary position of the dolphins. The dolphins during registration were producing various types of the signals freely, not suspecting at all, that they are registered at this time. The two-channel system of registration has advantage in comparison with a single-channel one because enables to restore spatial localization of sound sources and to give the qualitative evaluation of the signals directivity (as the result of processing the difference in levels and arrival times of the signal between the channels). Thanks to this the recorded signals had matched precisely with each dolphin, as well as all reflections of the signals from the edges of pool. Till now the acoustical signals of dolphins were not registered by a two-channel system.

The studying of characteristics and functions of the dolphins' acoustical signals is the purpose of the study. Specific tasks consisted in the recordings and subsequent analysis of the signals produced by two dolphins of one species by the two-channel recordings system in the pool conditions, in frequencies band of $0.1-220 \mathrm{kHz}$.

\section{Procedure}

Experiments were carried out on two adult Black Sea dolphins (Tursiops truncatus) with nicknames Yasha (male) and Yana (female), in the indoor pool of $23 \times 9 \times$ $4.5 \mathrm{~m}$ of Karadag Natural Reserve of NAS of Ukraine. The dolphins are in the pool about 20 years and have normal hearing.

The experimental configuration is shown in Figure 1(a). The experiment was carried out without special training of the dolphins. The time intervals when the dolphins approached near to the gangway (5) and remain on a water surface, almost without locomotion (Figure 1(b)) were used for recording their acoustical signals. The signals are recorded by the two-channel recording system. The matching of the registered signals with the dolphins as well as and the reflections of the signals from the edges of pool (the side walls, the bottom, the surface of water) were performed at the two-channel recording analyzing. For this purpose the signal arrival times difference and the levels difference of the given signal on the hydrophones of channels I and II as well as and known distances between the dolphins, the hydrophones and the edges of pool were taken into consideration.

The distance between the hydrophones (the base $3.5 \mathrm{~m}$ ) were chosen such to receive the necessary difference in levels and arrival times of each signal on the hydrophones, and to arrange them in the dolphin's far acoustic field (approximately $>1.5 \mathrm{~m}$ ). At the same time, the hydrophones were placed $1 \mathrm{~m}$ below the water surface in order to reduce the probability of signals shielding by the other dolphin in direction of the hydrophone distant to the dolphin producing sounds (Figure 1(a)). Besides, the hydrophones were located so that (2) was placed near to the pool sidewall whereas another one (1) further of the sidewall for signals reverberation evaluation in the pool.

The recordings were obtained by using the spherical hydrophones (1 and 2) with diameter of $14 \mathrm{~mm}$ and calibrated sensitivity $-203.5 \mathrm{~dB}$ and $-206 \mathrm{~dB}$ relative to 1 $\mathrm{V} / \mu \mathrm{Pa}$, or 66.5 and $50 \mu \mathrm{V} / \mathrm{Pa}$, respectively. The hydrophones' frequency characteristics have irregularity of $\pm 3 \mathrm{~dB}$ up to frequencies of $160 \mathrm{kHz}$ and $\pm 10 \mathrm{~dB}$ up to frequencies of $220 \mathrm{kHz}$. Each recording channel consisted of the hydrophone, the $0.1 \mathrm{kHz}$ high-pass filter, the $40 \mathrm{~dB}$ voltage amplifier and the one channel of the 14 bit analog-digital converter (USB-3000). The digitized signals of the dolphins during recording were continuously transferring from the analog-digital converter to the hard disk of notebook via interface USB 2.0. The analog- digital converter has dynamic range of $81 \mathrm{~dB}$ and sampling frequency of $1 \mathrm{MHz}$ for each channel. The frequency characteristic of the analog-digital converter at the given sampling frequency was flat up to $160 \mathrm{kHz}$ and smoothly 


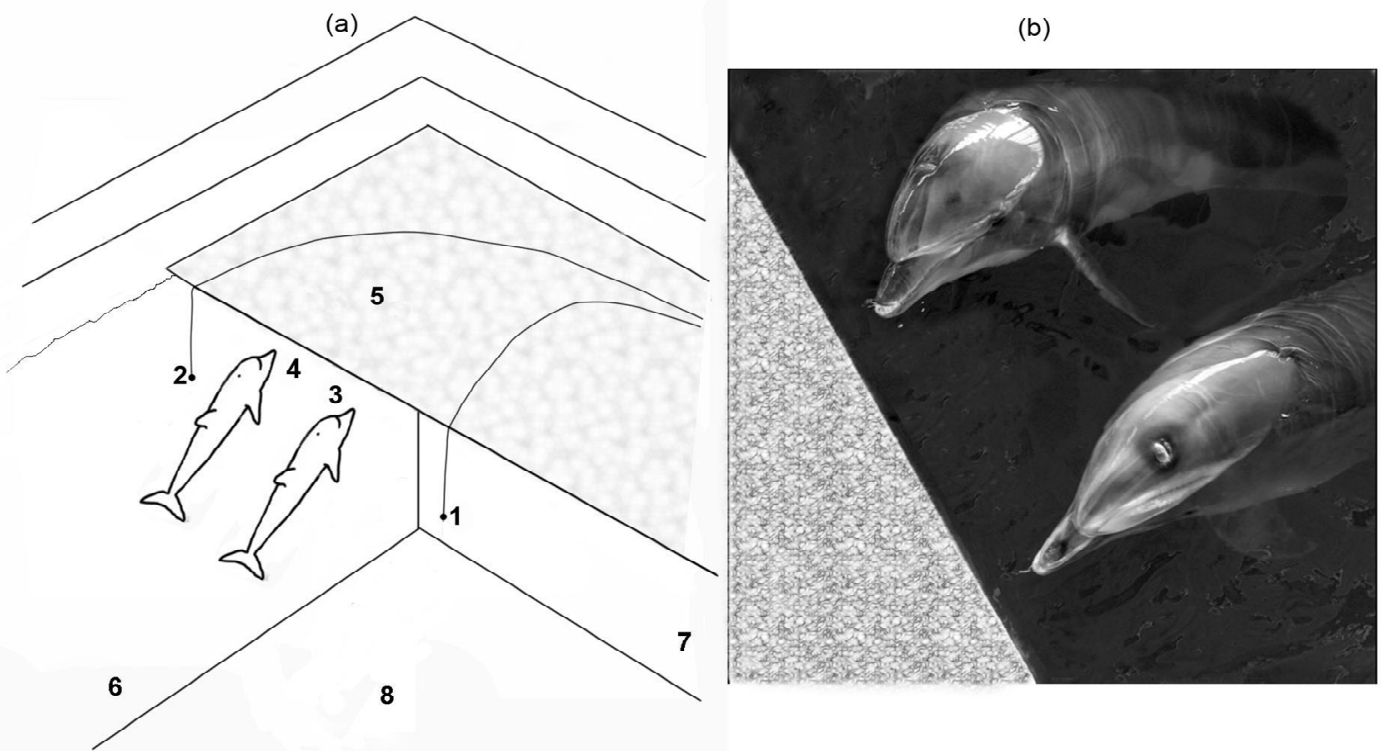

Figure 1. The experimental configuration. (a) 1, 2 are the hydrophones of channels I and II, respectively. The distance between hydrophones is $3.5 \mathrm{~m}$ at the depth of $1 \mathrm{~m} .3,4$ are the dolphins Yana and Yasha, respectively. The distance between dolphins is about $1 \mathrm{~m} .5$ is the gangway located $0.1 \mathrm{~m}$ above water surface. 6,7 and 8 are the long wall, short wall and bottom of the pool, respectively. The distance between the hydrophone 2 and sidewall 6 is $0.45 \mathrm{~m}$. The distance from hydrophones to the wall 7 is $3 \mathrm{~m}$. The water level is $4 \mathrm{~m}$. (b) The quasi-stationary position of the dolphins during the recording.

fell down to $-3 \mathrm{~dB}$ on frequency $200 \mathrm{kHz}$ for each channel. The programs packages of PowerGraph 3.3.8 and Adobe Audition 2.0 were using for recording and analyzing acoustical signals of the dolphins. The 4096-point FFT with Hamming windowing function or 1024-point FFT were used for the signals spectrums computing, Figure 2 and Figures 3-5, respectively. A lot of acoustical signals produced by the dolphins freely at will were recorded. The most typical recording (Figure 2) was chosen for the analysis and discussion. The dolphins were in quasi-stationary position (Figure 1(b)) in relation to the hydrophones and each other during the recording (Figure 2), approximately $34 \mathrm{sec}$. No other mammals were in the pool during the signals recording.

\section{Results}

The time dependence of all sounds produced by the dolphins during of $34 \mathrm{sec}$ of the recording and the sonogram of area with whistles from 3 th to 14 th sec are given in Figure 2. One can see that the dolphins produced the packs of pulses with the time intervals between the packs (789 - $4000 \mathrm{msec}$ ) more than the interpulse intervals (Table 1). The packs sequence in Figure 2 is designated by the numerals $(1-10)$. Duration of the pulses in the packs vary from $80 \mu \mathrm{sec}$ up to $600 \mu \mathrm{sec}$. The waveform of each pulse is complex (Figure 3) and varies from the pulse to the pulse in each pack. Therefore, the spectrum of each pulse as well varies from the pulse to the pulse (Figure 3(a)). In this connection, I have named their as the non-coherent pulses. The spectrum of the non-coherent pulses covered approximately all hearing range of a dolphin, from $6-15$ up to $160-200 \mathrm{kHz}$ and had many maximums and minima. However, considering that hearing thresholds of a dolphin steeply increase from frequencies above $135 \mathrm{kHz}$ the signals sonograms in Figures 3 and 5 are presented only up to $160 \mathrm{kHz}$. The sound pressure levels (SPL) of the non-coherent pulses vary from 15 up to $330 \mathrm{~Pa}$ (Figure 2). The number of pulses in the packs varies from 4 up to 27 pulses, but more often the packs have from 6 up to 8 pulses with the interpulse interval from $19 \mathrm{msec}$ up to $300 \mathrm{msec}$ (Table 1). The arrows near numerals (Figure 2(a)) specify the direction of pulses transfer, from Yasha to Yana or on the contrary.

Except the non-coherent pulses there were recorded also the packs of pulses with the waveform and spectrum that are invariable within each the pack but significantly vary from a pack to a pack (Figures 2-4). In this connection I named them as the coherent pulses. The SPL of the coherent pulses not exceed of $3-5 \mathrm{~Pa}$ what are about two orders lower of the SPL of the non-coherent pulses (Figure 3). In the Figure 2 these pulses are barely perceptible, thus the producer of the coherent pulses is designated by the conventional sign $n_{n}$ (where ${ }_{n}$ is the serial number of the pack) in the pack localization place, respectively. In the Figures $\mathbf{3}$ and $\mathbf{4}$ these pulses are shown in more convenient scale. The waveforms of coherent 
(a)
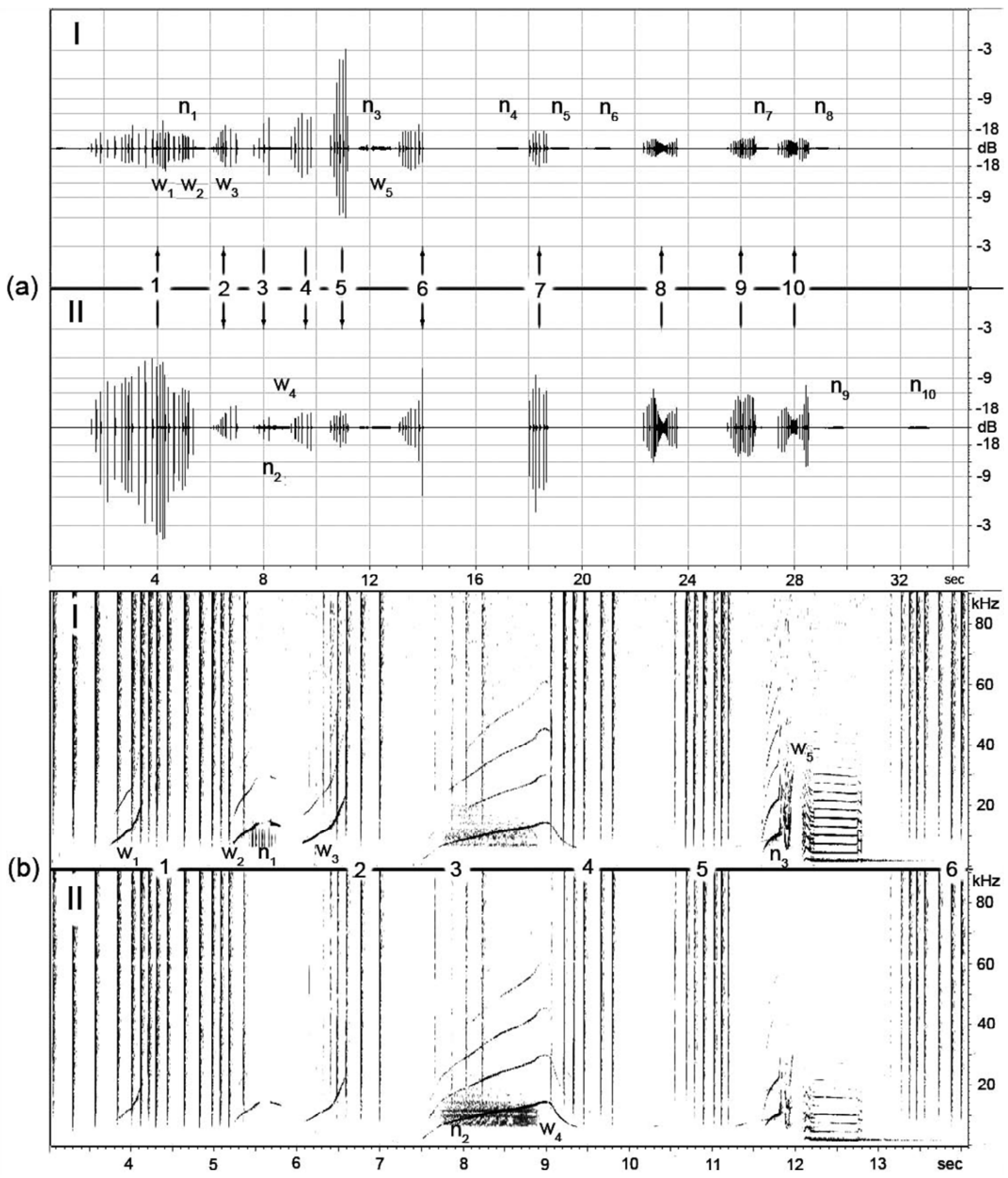

Figure 2. The time dependence of the dolphins' acoustical signals (a). The sonogram of area with whistles (b). Along X-axis are the signals locations on the time axis. Along Y-axis are SPL in dBs relatively $350 \mathrm{~Pa}$ and frequencies in $\mathrm{kHz}$, respectively. The numerals $(1-10)$ are the packs sequence. The arrows near the numeral specify the message transfer direction. $w_{n}$ and $n_{n}$ are localization nth whistles and nth packs of the coherent pulses, respectively. I and II are the channels numbers, respectively.

pulses are complex, the pulses duration was about 350 $650 \mu \mathrm{sec}$ (Figures 3(b) and 4). The spectrum of coherent pulses covered the frequencies band from $7-10$ up to 22 - $100 \mathrm{kHz}$ and had many maximums and minimums.

Copyright (C) 2011 SciRes.
There were the packs of the relatively narrow-band and broadband pulses. The interpulse interval in packs of coherent pulses always varies, as a rule gradually decreasing from the beginning to the end of a pack and is 
Table 1. The characteristics of the non-coherent pulses' packs.

\begin{tabular}{ccccccccccc}
\hline pack number & 1 & 2 & 3 & 4 & 5 & 6 & 7 & 8 & 9 & 10 \\
\hline $\begin{array}{c}\text { pulses numbers } \\
\text { in pack }\end{array}$ & 23 & 8 & 4 & 6 & 7 & 8 & 6 & 27 & 14 & 19 \\
$\begin{array}{c}\text { interpulse } \\
\text { interval (msec) } \\
\text { pack duration } \\
\text { (msec) }\end{array}$ & $90-266$ & $80-230$ & $180-240$ & $160-212$ & $89-136$ & $87-181$ & $95-170$ & $19-126$ & $68-112$ & $31-138$ \\
& 3850 & 860 & 580 & 770 & 620 & 890 & 620 & 1250 & 1100 & 1120 \\
\hline
\end{tabular}

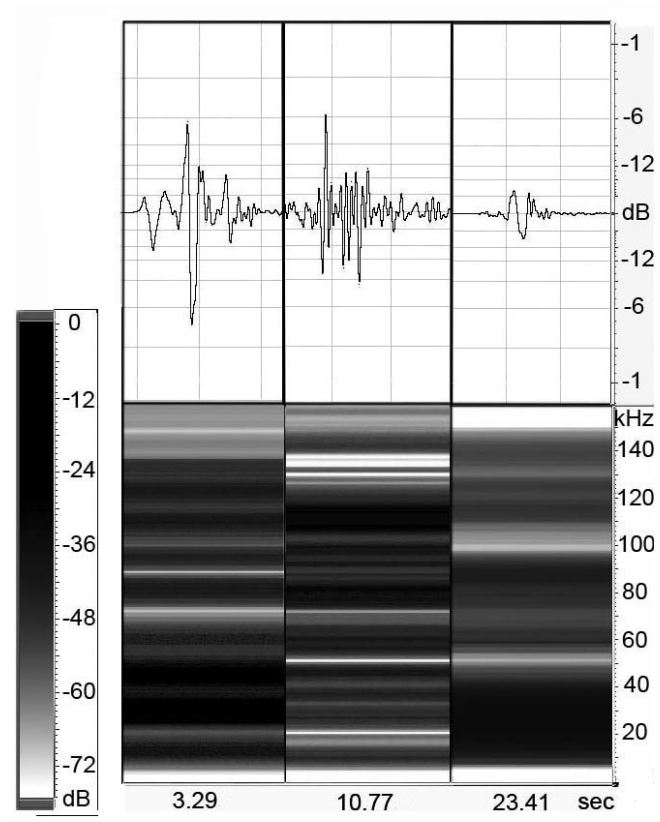

(a)

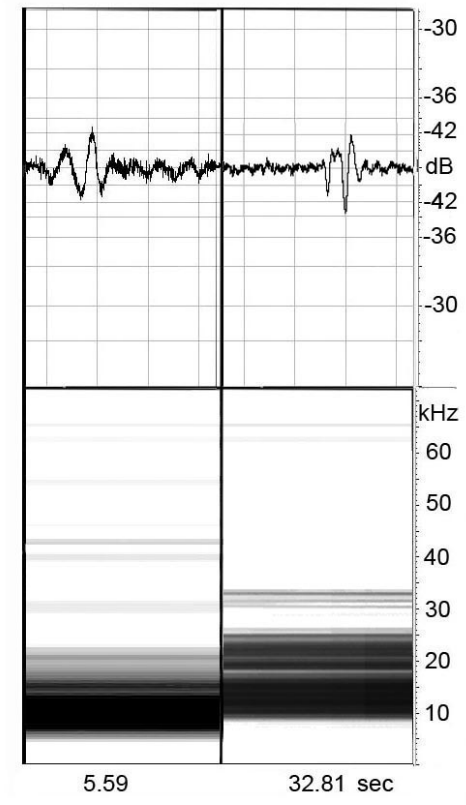

(b)

Figure 3. The non-coherent (a) and coherent (b) pulses of the dolphins in the time (above) and frequency (underneath) domain. Along X-axis are signals locations on the time axis of Figure 2. Along Y-axis are SPL in dBs relatively 350 Pa, the relative amplitudes of sonogram in $\mathrm{dBs}$ and frequencies in $\mathrm{kHz}$, respectively. The time scale for the pulse produced on 32.81 th sec is $200 \mu \mathrm{sec} / \mathrm{div}$, and $100 \mu \mathrm{sec} / \mathrm{div}$ for the rest.

seldom on the contrary (Figure 4(a), Table 2). The relative variation of interpulse interval was within of 1.1 5.6 and not depended from duration of a pack. The packs contain from 20 up to 611 pulses with the interpulse interval within of 1 - $41 \mathrm{msec}$ (Table 2).

The dolphins produced five frequency-modulated whistles with harmonics up to $100 \mathrm{kHz}$ (Figure 2(b)). The whistles producer is designated by the conventional sign $w_{n}$ (where ${ }_{n}$ is the serial number of whistle) in the whistles localization places, respectively. Fundamental frequency of the whistles (Figure 2(b)) vary in the band of $8-21 \mathrm{kHz}, 8-16 \mathrm{kHz}, 8-22 \mathrm{kHz}, 3-16 \mathrm{kHz}$ and $2.8-28$ $\mathrm{kHz}$, and the number of harmonics on the hydrophone placed near to whistling dolphin were of 1, 2, 1, 4 and about 16, from first to fifth whistle, respectively. On the distant hydrophone from whistling dolphin the harmonics were absent at first three whistles, at fourth whistle their quantity was the same, at the fifth whistle were about 7 harmonics. On account of relatively low SPL of the whistles and coherent pulses their reflections from the sidewalls and bottom of the pool, apparently, were masked by noise and are imperceptible.

At the same time the reflections of the non-coherent pulses are well visible both in the time and frequency domain (Figure 5). On account of the dolphins' quasistationary position the reflections locating of the each non-coherent pulse from pool edges approximately are identical and for the example is given in Figure 5 for the pulse that Yana produced on 10.88th sec.

\section{Discussion}

Dolphins specially were not trained to produce acoustical signals in this experiment. Besides, their position on a 
(a)

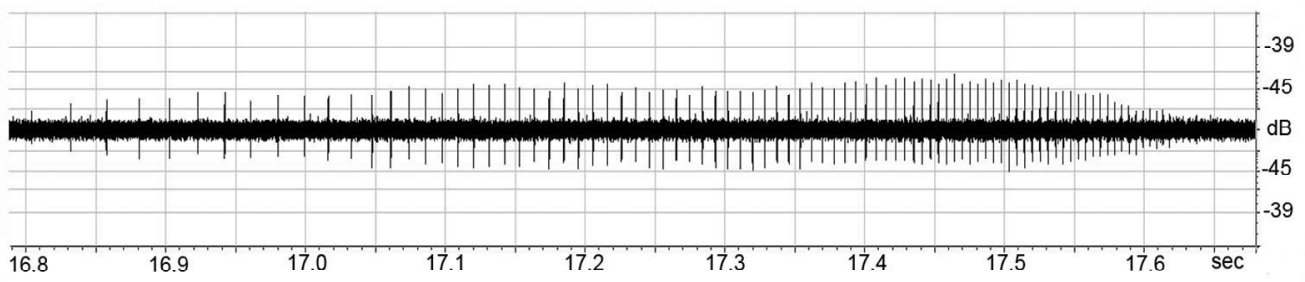

(b)

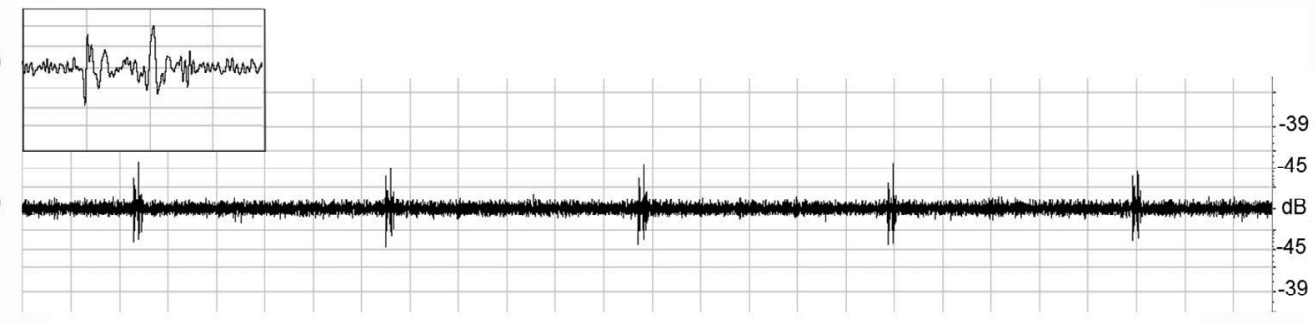

(d)

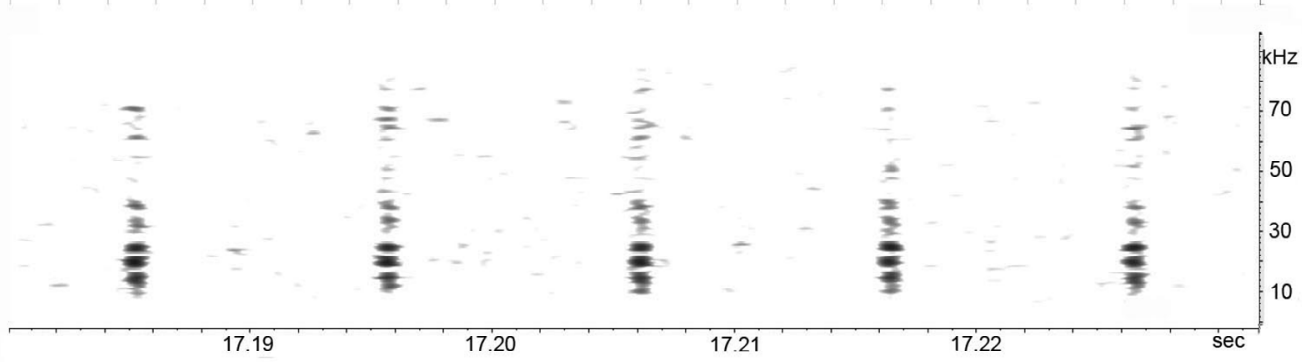

Figure 4. Example of the time dependence of interpulse interval in the pack of coherent pulses (a) that Yana produced from 16.8th up to 17.6th sec $\left(n_{4}\right.$, Figure 2$)$ and a magnification of the area from 17.185th up to 17.23th sec represented in the time (c) and frequency (d) domain. The single pulse (b) at the expanded time scale of $200 \mu \mathrm{sec} / \mathrm{div}$. Along $\mathrm{X}$-axis are pulses location on the time axis of Figure 2(a). Along Y-axis are SPL in dBs relatively $350 \mathrm{~Pa}$ and frequencies in $\mathrm{kHz}$, respectively. The relative amplitudes scale of sonogram is the same as in Figure 3.

Table 2. The characteristics of the coherent pulses' packs. In the row "interpulse interval" the first value is the interpulse interval in the pack beginning, the second value - in the end.

\begin{tabular}{ccccccccccc}
\hline pack number & 1 & 2 & 3 & 4 & 5 & 6 & 7 & 8 & 9 & 10 \\
\hline $\begin{array}{c}\text { pulses } \\
\text { numbers in pack }\end{array}$ & 24 & 611 & 20 & 86 & 73 & 56 & 119 & 72 & 202 & 104 \\
$\begin{array}{c}\text { interpulse interval } \\
\text { (msec) }\end{array}$ & $8.5-6$ & $2-1.8$ & $1-2.5$ & $28-5$ & $13-5$ & $19-6.5$ & $41-13.5$ & $15-4$ & $9.5-3.8$ & $29.5-5.7$ \\
$\begin{array}{c}\text { relative variation of } \\
\text { interpulse interval }\end{array}$ & 1.4 & 1.1 & 2.5 & 5.6 & 2.6 & 2.9 & 3 & 3.7 & 2.5 & 5.2 \\
pack duration (msec) & 300 & 1100 & 40 & 850 & 820 & 505 & 670 & 410 & 758 & 822 \\
& & & & & & & & & &
\end{tabular}

water surface almost without locomotion (Figure 1(b)) more likely speaks to an outside observer that they dozed. However, when the two-channel recording system was turned on it was discovered that the dolphins at this time carry on the lively conversation using the main arsenal of signals communication (Figure 2). This means that after 20 -years of theirs staying in the pool the dolphins still have what to tell to each other!

The dolphins did not produce outgoing pulses (clicks) of the sonar with rostral directivity [24] during this recording (Figure 2), probably, in it was not necessities; therefore their melons were partially above water.

Copyright (C) 2011 SciRes.
Though, the standard stereotype acoustical clicks with duration approximately $25 \mu \mathrm{sec}$ and the spectrum maximum near of $115 \mathrm{kHz}$ were recorded during other recording when the dolphins were under water. It is necessary to note, that SPLs both the non-coherent and coherent pulses were by one-two orders less of the sonar's clicks levels. Besides, each type of the pulses mostly had characteristic waveforms.

All recorded pulses (Figures 2-5) apparently are the acoustical signals that widely considered as the dolphins' social signals [13-21]. However, in these works the packs of pulses were studied in the frequency band up to 

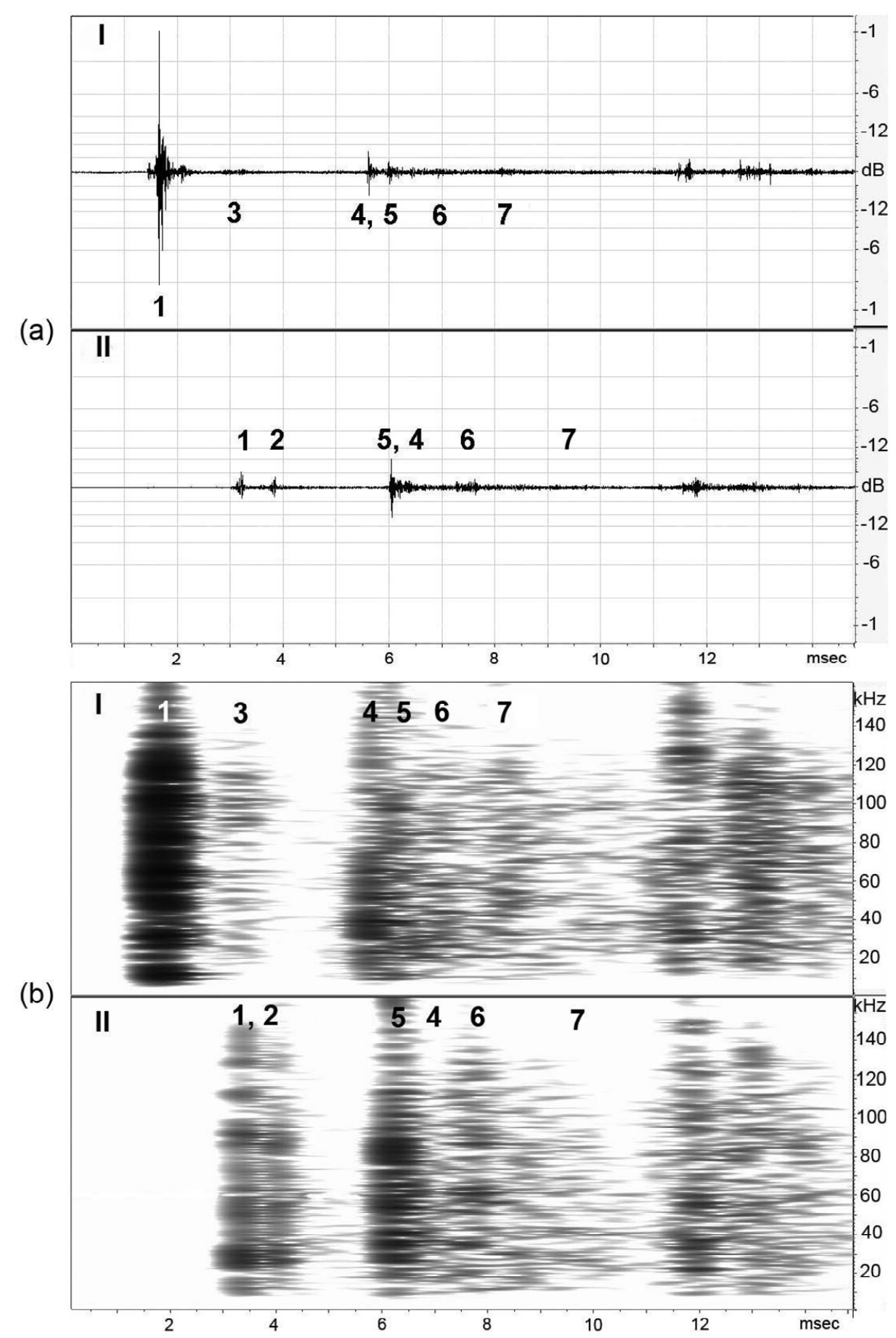

Figure 5. The non-coherent pulse that Yana produced on 10.88-th sec (Figure 2) and the pulse reflections in the time (a) and frequency (b) domain. 1 is the non-coherent pulse. 2 is the echo from wall (6, Figure 1). 3 is the echo from dolphin Yasha. 4 is the echo from wall (7). 5 is the echo from pool bottom (8). 6 is the double echo: the bottom of pool-the surface of water. 7 is the echo from wall opposite to wall (6). Along $\mathrm{X}$-axis is the time in msec. Along $\mathrm{Y}$-axis are SPL in dBs relatively $277 \mathrm{~Pa}$, and frequencies in $\mathrm{kHz}$, respectively. The relative amplitudes scale of sonogram is the same as on Figure 3.

$20 \mathrm{kHz}$, therefore it is difficult to compare the obtained results (Figures 2-5) with the literature data. In the same time the recorded pulses have too low energy levels on Copyright (C) 2011 SciRes. frequencies of human hearing and at playing back the record as a result there are only weakly audible the pulses-repetition frequencies. Therefore, to classify the 
pulses aurally as: “quack", “squeak”, “squawks”, "bark”, "squeal", etc. [16] it turns out practically impossible. In this connection, apparently, it is necessary to agree with the opinion stated in the work [22], and to reconsider classification of dolphins' signals in view of their physical characteristics. Therefore in the present work the recorded pulses are described mostly from the point of view of their physical characteristics.

In total the dolphins had produced 122 the noncoherent pulses in 10 packs, 1465 the coherent pulses in 10 packs and 5 the whistles, for 34 sec! All recorded whistles (Figure 2) contained harmonics quantity more on the hydrophone nearest to whistled dolphin. In general, the types of fundamental frequency contours of whistles and the harmonics quantity are typical for these animals [25-29].

The characteristics of the coherent pulses (Figures 3 and 4, Table 2) are practically completely coincide with the burst pulses registered in free-ranging the Hawaiian spinner dolphin (Stenella longirostris) and the Atlantic spotted dolphin (Stenella frontalis) [22,23]. Moreover, the dolphins Yana and Yasha gradually controlled the interpulse interval value in each pack (Figure 4 and Table 2), and in the different degree. The relative changes of the interpulse interval were from 1.1 up to 5.6. The interpulse intervals in the packs of coherent pulses always vary, as a rule, gradually decreasing from the beginning to the end of a pack and are rare on the contrary. In general, the value control of interpulse interval also it is possible to consider as control of the phase of each subsequent pulse in the pack.

The dolphins in each case used the necessary quantity of pulses (from 20 up to 611) with the necessary interpulse interval (from 41 up to $1 \mathrm{msec}$ ) (Table 2). Besides, the dolphins used pulses of the necessary waveform and spectrum in each pack. Spectrum of the coherent pulses of Yana and Yasha also as and of Hawaiian (Stenella longirostris) and Atlantic (Stenella frontalis) dolphins, had many maximums and minima and covered the frequencies band up to $100 \mathrm{kHz}$. The spectral maximums and minima in each pack as well as and the pulses waveform did not vary in the pack but ones significantly vary from the pack to the pack (Figures 3 and 4). Major energy of the coherent pulses is concentrated on frequencies above $7-10 \mathrm{kHz}$, and there were packs of relatively narrow-band and broadband pulses as in work [22]. Hence, Black Sea dolphins (Tursiops truncatus) produced the packs of coherent pulses (Figures 2-4) just like and the Hawaiian and Atlantic dolphins [22,23].

The locating of all dolphins signals in the time domain has character of an exchange of three types of signals, Figure 2(a). The signals of each type each dolphin has produced in turn, without the overlapping in time. For the whistles produced by Yana (Figure 2, $\mathrm{w}_{1}-\mathrm{w}_{3}$ ) Yasha did answer with the whistle $\left(\mathrm{w}_{4}\right)$, after that the whistle of Yana $\left(w_{5}\right)$ follows again. In the same way, without overlapping in time, the dolphins do exchange packs of the coherent pulses (Figure 2, $\mathrm{n}_{1}-\mathrm{n}_{10}$ ). In total, Yana produced 7 packs of the coherent pulses (548 pulses) and Yasha 3 packs of the coherent pulses (917 pulses).

The dolphins exchange packs of the non-coherent pulses in a more complicated manner (Figure 2). There is obvious levels distinction of each pulse between channels I and II. Yana is located (Yasha is located) during all record nearer to hydrophone of channel I (II), therefore on channel I (II) all pulses which she (he) produces have level higher. The packs of non-coherent pulses $(2,3$, 4 , and 6, Figure 2) have less appreciable distinctions of the levels between channels I and II, therefore the producers of these pulses have been identified in view of the difference in the pulses arrival time on the hydrophones. In the pack (2) both dolphins produced pulses almost simultaneously and therefore, it is possible to assume, that they not understood each other, therefore Yasha has conceded and has allowed the lady to express oneself (packs of 3 - 5). In the pack (6) again, both dolphins almost simultaneously produced pulses, and though Yasha gradually increased their level, from the pulse to the pulse, Yana, apparently, again not listened him, then he loudly shout (last pulse in the pack 6). Then, after the short pause Yasha produced the packs of pulses (7 - 10) already up to the end of the record, having lowered the loudness of voice (the packs of 8 - 10).

The signals of different type could be produced by each dolphin simultaneously (Figure 2). So, Yana simultaneously produced as the coherent pulses and whistles from 5.4th to 5.8th sec and from 11.9th to 11.95th sec, so and the non-coherent pulses and the coherent pulses from 26.26th to 27th sec. Yasha produced simultaneously the non-coherent pulses, coherent pulses and whistles from 7.6th to 9th sec. These facts testify that each type of the signals was produced independently by the appropriate organ of dolphins. Hence, in view of the sonar clicks of a dolphin [24], apparently, in a dolphin should be at least four the organs that independently produce four types of signals.

Inasmuch as in the pool there were no other animals and the dolphins nobody forced to produce any sounds, it is possible to believe, that dolphins produced all these sounds or for communication with each other, or for orientation in the pool in spite of daylight (i.e. to feel the moving concerning each other, the walls and bottom of pool; to monitor ambient environment for distance more than water transparency). In what degree each type of the recorded signals can be used by the dolphins for the communications and orientation? 
The non-coherent pulses produced by the dolphins differ from each other by the waveform in time domain and by the set of spectral components in frequency domain (Figure 3). In this connection it is possible to assume, that each non-coherent pulse represents the phoneme of the speech of a dolphin spoken language and every pack of the non-coherent pulses is a word, then sequence of the packs of the non-coherent pulses is the sentence. For the best understanding, we shall compare speech of a dolphin spoken language with speech of a human spoken language. In dolphins the phonemes spectrum covers almost all frequencies band of a hearing, from $6-15 \mathrm{kHz}$ up to $160 \mathrm{kHz}$. Frequencies approximately below $6-15 \mathrm{kHz}$ (Figure 2(b)), apparently, are excluded from a dolphin speech for increasing a speech noise-immunity, because for the frequencies below approximately $10 \mathrm{kHz}$ both a dolphin's hearing thresholds and a level of environmental noise significantly increase. Phonemes spectrum of a human speech also covers almost all frequencies band of a human hearing, but approximately $0.3-4 \mathrm{kHz}$ is necessary and sufficient for speech intelligibility; however this frequencies band is located primarily beyond coverage of a dolphin speech. The duration of dolphins phonemes are relatively very short, $80-600 \mu \mathrm{sec}$, that on $2-3$ orders shorter than duration of a human phonemes. In addition, between dolphins phonemes there are relatively long-duration interpulse intervals (the characteristic value nearby of $150 \mathrm{msec}$ ). This interval can widely vary and, apparently, is necessary for phonemes immunity from reverberations. The phonemes of a human spoken language also consist of spectral components, but as opposed to a dolphin a human is producing phonemes of one word inseparably, as a solid word. In this connection duration of a dolphin word and a human word can be approximately identical at identical quantity of phonemes. Probably, in the free-ranging dolphins the interpulse interval will be considerably less in this case words duration will decrease.

Certainly, it is hardly likely, but every the non-coherent pulse of a dolphin may be represents the word and a pack of pulses represents the sentence, but at present this is inessential as the answer to this question will be given by the further researches.

It is possible to estimate in what degree the spoken language of a dolphin possesses the Hockett's 13 design features of human language [30]. The design features we will consider in the order, like they were introduced by author.

1th - 6th of the design features, obviously, are inherent for the dolphin and, apparently, do not demand special discussion. It is more interesting to consider from the 7th to 13th features because ones in a significant extent are determined by an intellect and level of an animal's con- sciousness.

7) Semanticity. It were shown experimentally [31,32] that dolphins understood novel instructions conveyed within artificial gestural or acoustic language systems using "sentences" as long as five words whose interpretation required processing of both the semantic and syntactic features of the languages. That maybe present indirect confirmation that and in the spoken language of a dolphin a specific signal can be matched with a specific meaning.

8) Arbitrariness. Concept learning was demonstrated within artificial gestural or acoustic language systems within several paradigms, including discrimination learning sets and matching-to sample [33]. This is indirect confirmation that and in the spoken language of a dolphin there is no limitation to what can be communicated about and there is no specific or necessary connection between the sounds used and the message being sent.

9) Discreteness. In the spoken language of a dolphin the phonemes discreteness (Figure 3) is determined by the different locating on frequency and level of the maximums and minima of spectral components. It is easy to notice, that these distinctions more than differential thresholds of a dolphins' hearing on frequency-about $0.2 \%-0.8 \%$ in a frequencies band of $10-130 \mathrm{kHz}$ [34-36] and on intensity-about $10 \%$ [37,38].

10) Displacement. It was shown that the dolphin understood gestural instructions within artificial gestural language system as reliably when conveyed through television images of trainers as when conveyed by live trainers. The words of that language were understood referentially, including an ability to report whether a referenced object was present or absent in the dolphin's tank $[33,39]$. It attested that a dolphin have ability to refer to things in space and time and communicate about things that are currently not present and indirectly testifies that a dolphin have the high level of consciousness and may have the highly-developed language.

11) Productivity. It is possible to estimate approximately what quantity of words can to be created from various combinations of separate spectral components in the spoken language of a dolphin. If at analysis of the pulse spectrum (i.e. the phonemes) the dolphin uses the mechanism of critical bands with about $10 \%$ width from the central frequency of the hearing filter [40-43] then in the frequencies band of $10-130 \mathrm{kHz}$ about 15 critical band are located. We shall assume, each phoneme consists of 10 spectral components (10 spectral strips of Figure 3). Then the quantity of different phonemes can be equal to the number of permutation of 10 out of 15 , without recurrences that give nearby $3 * 10^{3}$. This is obviously excessive. If the dolphin spoken language uses 
by analogy to human spoken language even 30 different phonemes from them it will be possible to create quantity of words comparable to productivity of a human language for which, as it is known, this value is defined by number with 26 zero. In what degree the spoken language of a dolphin is opened, it will be possible to tell, apparently, only at further studying of the language.

12) Traditional transmission. Nongenetic transmission of social behavior across generations was observed for Cetaceans [44,45], it suggest that, these large-brained, slow-developing, and socially complex species [46] have evolved powerful social-learning mechanisms. Some potential scenarios and mechanisms observed for a group of free-ranging Atlantic spotted dolphins (Stenella frontalis), including implications of vertical, horizontal, and oblique directions of transmission of social information during various behavioral contexts are described [47]. Teaching, then, may be an important way in which aspects of cetacean social learning and possibly culture are transmitted from one generation to the next [48]. These outcomes suggests while certain aspects of language may be innate, dolphins maybe acquire words and their native language from conspecifics.

13) Duality of patterning. On the basis that the dolphins' spoken language consist from phonemes it is possible to assume that and dolphins have ability to recombine a finite set of phonemes to create an infinite number of words, which in turn can be combined to make an unlimited number of different sentences.

Thus, the spoken language of the dolphins, expressly or by implication, conforms to all the design features of a human language, and it is possible to consider as the highly-developed language. In favor of this as well indicate the facts that dolphins possess large and highly developed brain already more than twenty million years [12].

But, unfortunately, the frequency-time characteristics of a dolphin speech are localized outside limits of a human hearing, therefore a dolphin speech exist beyond reach of a human hearing. In contrast to it the frequencytime characteristics of a human speech are localized within the area of a dolphin hearing, nevertheless as a result of significant reflection of sound energy on air-water interface, a dolphin hear an attenuated human speech. In this connection, for an establishing of mutual relations with these intellectual mammals a human should take the first step should create a device capable to overcome the physical restrictions for use of both languages. It should like to think, that dolphins will tell the last word at decoding the languages because they seemingly can possesses even higher forms of consciousness than human.

As it follows from the record (Figures 2 and 5) the echo-signals of the non-coherent pulses both from sidewalls and pool bottom and even from dolphin Yasha have the high levels. In the other words the echo-signals levels from rostral, lateral and ventral directions of the dolphins are approximately identical. Hence, these pulses have no directivity and play a role of both the dolphin speech phonemes and the probing pulses of the non-coherent dolphin sonar which necessary for near orientation in coastal conditions of shallow water and rocks, and also for orientation among floating relatives. Meanwhile, rather small phonemes duration and rather wide interval between phonemes define high time resolution and anti-reverberation immunity of the dolphin speech. The mean values of interpulse interval in the packs of the non-coherent pulses on the record (Figure 2) were about $150 \mathrm{msec}$, that give the sonar range indirect assessment at least about $112 \mathrm{~m}$ in view of the two-way of a sound up to the target. The length of our pool is $23 \mathrm{~m}$, so the dolphin produced every pulse on the average after the fivefold echo along the pool from previous.

Absence of the directivity non-coherent pulses is caused due to natural necessity in broadcast transmission both the phonemes and probing pulses. Whereas the directional reception both the echo-signals and dolphin speech from all directions around of a dolphin can be performed by a new external ear [8-11] with the localization accuracy of $1^{\circ}-3^{\circ}$ [49-51].

Hence, the non-coherent pulses are of great importance both for dolphins' communication as the speech, and for orientation as the probing pulses of non-coherent sonar, i.e. the pulses are bimodal.

What significance may have the whistles and coherent pulses for communications and orientation of dolphins? The major energy of whistles coincides with frequencies band of human hearing, therefore a lot of works is devoted to studying of these signals and has been stated more hypotheses, in comparison with other types of dolphins acoustical signals. Large repertoires of the context-specific whistles that vary according to situation or activity were described and discussed $[25,26,52]$. In addition the whistles can presumably be used by dolphins as the signature-whistle identifying each dolphin among the others as individually distinctive according to the whistle contour [27]. Besides, the whistles can play an important role in maintaining contact between dispersed conspecifics [28], and also for definition of a direction of movement of a whistling dolphin [24,53]. The calculated range for dolphins' communication by whistles corresponds approximately of $10.5 \mathrm{~km}$, for whistles with highest (167 dB re. $1 \mu \mathrm{Pa})$ source level [29].

It is interesting that whistling obviously is not used in the human speech. At the same time, even not realize it, the human easily can whistle from surprise, can whistle 
when good mood, or to whistle loud from far away in order that to attract attention. The whistle can be transmitted on farther distance than a speech because one has a lot of energy in the narrow frequencies band. Our dolphins, Yana and Yasha apparently know this because they lift up the heads out from water and can whistle in air, specially, in order that to draw attention of a human. Thus they emit very loud whistle with the lowest fundamental frequency $(2.8 \mathrm{kHz}$, practically without frequency modulation, Figure 2, second part of the whistle $\mathrm{w}_{5}$ ) which are audible in the most remote corners of our dolphinarium.

However by analogy with the non-coherent pulses it is possible to assume, that dolphins can use whistles not only as communication signals but also for orientation. From the point of view of physical acoustics the whistles can be used in the FM-Doppler sonar. In engineering applications such sonar is used for detecting moving targets or for measuring the relative radial velocity of target and for range measurement. The Doppler frequency shift $f_{D}$ depend on the carrier wavelength $\lambda$ and the relative radial velocity (or range rate) between the sonar and the target $V r$, as: $f_{D}=-2 V r / \lambda$, where $\lambda=c / f$ is carrier wavelength, $c$ is the sound velocity in water, $f$ is the carrier frequency. Hence, with increase of carrier frequency (of fundamental frequency of a dolphin whistling) the sonar wavelength will decrease and so the Doppler frequency shift will increase that will increase the sensitivity of the sonar. Meanwhile, the frequencies of whistles harmonics increase proportionally to the number of harmonic. If dolphins do analyzing the echo of whistles harmonics, then thereby they increases sensitivity of sonar proportionally to the number of harmonic. That in some degree explains availability of plenty harmonics in dolphins whistles and confirms possibility the harmonics using by Doppler sonar of dolphins. The maximum sensitivity of Doppler sonar can be necessary to dolphins on small distances when it is important the detection of small relative radial velocities and small relative movings which the sonar cannot perceive on the fundamental.

Besides it is known, that in engineering applications the sonar with pulse compression uses a long tonal pulse with linear or nonlinear frequency modulation similar to the contours of fundamental frequency of a dolphin whistles. If a FM pulse of this sonar has a spectrum width $B$, and the returned echo is processed by the matched filter with a pulse compression then the pulse response duration will be approximately $1 / B$ and will not depend on the duration of the probing pulse. The more a spectrum width of a pulse, the less a filter reaction duration of the receiver and in the result it is enhance the range resolution of sonar. In other words, such sonar receives an echo with energy as from a long pulse, but with the range resolution as from a short pulse. Therefore when the du- ration of whistle will increase then whistle energy will increase proportionally and the dolphin can receive echosignals of conspecifics from greater ranges and with the higher range resolution. It is interesting to note, that at maintenance of contact between the separate dispersed conspecifics, dolphins, apparently, can feel each other and actively by means of the FM-Doppler sonar (when a dolphin is whistling and analyzing echo of each conspecific) and passively (just analyzing the whistling of each conspecific), in this case the maximum range for exchanges of whistles in dolphins can reach up to $10.5 \mathrm{~km}$ [29]. Hence, whistles also as well as and the non-coherent pulses, apparently are bimodal, and ones can be used by dolphins, at the limited information exchange [24$29,52,53]$ as communication signals and for orientation as a probing signal of the FM-Doppler sonar with a pulse compression.

Dolphins produce the coherent pulses primarily on close distances to each other up to 3 - $14 \mathrm{~m}$, presumably for exchange of emotional signals [23], and the characteristics of the coherent pulses suggest that dolphins could use them as acoustical communication signals. However, the quantity of the coherent pulses produced by the dolphins in one pack up to 611 (Table 2) and even 958 according to [22], with gradually varying interval interpulse (or of the phase interpulse), and the coherence of the pulses in each pack, testifies more likely about utilization of the coherent pulses by the dolphins for orientation. As well, the spent time for transfer of the same pulse many times (Figure 4) is obviously not proportionally to the quantity of information in this pulse that testifies about the same [54]. But in the literature so far is not clear with what purpose dolphins use these pulses packs, thus additional researches are necessary.

However, from the point of view of acoustics the characteristics of the coherent pulses (Figures 3 and 4) completely conform to the signals of the pulse-Doppler sonar with high pulse repetition frequency. In engineering applications such sonar are using packs of coherent pulses with time intervals between the pulses many times less than the two-way time of a sound up to the target and where there is a fixed or deterministic phase relationship between each successive pulse, i.e. exactly as in the packs of the dolphins' coherent pulses (Figure 4, Table 2). Such sonar uses Doppler frequency shift for selection of the moving target in a severe clatter environment and radial velocity measuring. It is interesting to note, that the most studied sonar of a dolphin with a rostral directivity [24] is using the pulses (the clicks) with stereotype waveform, i.e. these pulses are coherent. But the pulses repetition frequency low so, that a time interval between pulses as a rule is more than two-way time of a sound up to the target. In engineering applications similar pulses 
uses sonar that have been categorized as the moving targets indication (MTI). The MTI selects the moving target in a severe clatter environment and measures distance up to the target. Both pulse-Doppler sonar and the MTI use the coherent transmission, reception and processing of echo-signals with the purpose to reject main-beam clutter and enhance target detection and aid in target discrimination or classification. Hence, the sonars with the coherent pulses dolphins in general are used for orientation. But considering, that all acoustical signals are being processed by the same hearing analyzer of the dolphin, apparently, these pulses can be used by dolphins both for transfer of the limited quantity of the information during social interaction of dolphins and for orientation as the pulse-Doppler sonar and as the MTI.

\section{Conclusions}

From findings of this work follows:

1) The echo-location system of dolphins and, apparently, Odontoceti is more complex than it was being discussed earlier, and it is very likely, the system has at least four various types of sonars with different types of the probing signals and with various methods of processing of the acoustical information.

2) There is a good reason to consider, that dolphins, and apparently, Odontoceti have the highly-developed spoken language. These findings confirm the representation about Cetacean as about the first reasonable animals of a planet the Earth.

3) The Nature has shown the ingenuity miracles already about 25 million years ago at creation of the echo-location system and spoken language of a dolphin. Hence the signals characteristics, obviously, are defined by their functionality and are optimum from the point of view of the modern state of physical acoustics.

The aspects considering of characteristics and possible functions of the dolphins signals in this study does not claim to be complete, but probably point out the perspectiveness of their further studying.

\section{Acknowledgments}

The author would like to thank Nikolay Bibikov (Andreev Acoustical Institute, Moscow) for the ADC (USB-3000) granting. Also, the personnel of the bioacoustics laboratory of sea mammals of Karadag Natural Reserve, especially to trainers Sveta Yahno and Nadya Zhukova for their invaluable assistance.

\section{References}

[1] R. Kellogg, "A Review of the Archaeoceti," Carnegie Institution of Washington Publication, Washington, D.C., No. 482, 1936, pp. 1-366.
[2] L. G. Barnes, and E. Mitchell, “Cetacea,” In: V. J. Maglio and H. B. S. Cooke, Eds., Evolution of African Mammals Harvard University Press, Cambridge, 1978, pp. 582-602.

[3] R. E. Fordyce, "Whale Evolution and Oligocene Southern Ocean Environments," Paleogeography, Paleoclimatology, Paleoecology, Vol. 31, 1980, pp. 319-336. doi:10.1016/0031-0182(80)90024-3

[4] P. D. Gingerich, N. A. Wells, D. E. Russell and S. M. I. Shah, "Origin of Whales in Epicontinental Remnant Seas: New Evidence from the Early Eocene of Pakistan," Science, Vol. 220, No. 4595, 1983, pp. 403-406.

[5] S. Leatherwood, R. R. Reeves, W. F. Perryn and W. E. Evans, "Whales, Dolphins, and Porpoises of the Eastern North Pacific and Adjacent Waters," NOAA Technical Report NMFS Circular, Vol. 444, 1982, pp. 1-245.

[6] W. A. Watkins, and D. Wartzok, "Sensory Biophysics of Marine Mammals," Marine Mammal Science, Vol. 1, No. 3, 1985, pp. 219-260.

[7] G. S. Miller, "The Telescoping of the Cetacean Skull," Smithsonian Misc Coll. No. 76, 1923, pp. 1-67.

[8] V. A. Ryabov, "Mechanisms of a Dolphin's Echolocation Hearing," In: S. Dible, P. Dobbins, J. Flint, E. Harland and P. Lepper, Eds., (Bio-Acoustics 2007), Proceedings of the Institute of Acoustics, Vol. 29, No. 3, 2007, pp. 283-293.

[9] V. A. Ryabov, "Acoustic Clutter Field and Echo Reception by the Dolphin," Biophysics, Vol. 53, No. 3, 2008, pp. 237-242.

[10] V. A. Ryabov, "Role of the Mental Foramens in Dolphin Hearing," Natural Science, Vol. 2, No. 6, 2010, pp. 646653.

[11] V. A. Ryabov, M. A. Polyakov and N. G. Bibikov, "Characterization of Beam Patterns of Bottlenose Dolphin in the Transverse Plane," Biophysics, Vol. 56, No. 3, 2011, pp. 529-534.

[12] S. H. Ridgway, "Physiological Observations on the Dolphin Brains," In: R. J. Schusterman, J. Thomas and F. G. Wood Eds., Dolphin Cognition and Behavior, a Comparative Approach, Erlbaum, Hillsdale, 1986, pp. 31-59.

[13] J. C. Lilly and A. M. Miller, "Sounds Emitted by the Bottlenose Dolphin,” Science, Vol. 133, No. 3465, 1961, pp. 1689-1693.

[14] M. C. Caldwell and D. K. Caldwell, "Intraspecific Transfer of Information via the Pulsed Sound in Captive Odontocete Cetaceans," In: R. G. Busnel Ed., Animal Sonar Systems: Biology and Bionics, Laboratoire de Physiologie Acoustic, Jouy-en-Josas, France, 1967, pp. 879-936.

[15] P. Tyack, "Patterns of Vocalization in Wild Tursiops Truncates," Senior Thesis, Rockefeller University, 1976.

[16] L. M. Herman, and W. N. Tavolga, "The Communication Systems of Cetaceans," In: L. M. Herman, Ed., Cetacean Behavior: Mechanisms and Functions, Wiley Interscience, New York, 1980, pp. 149-209.

[17] A. N. Popper, "Sound Emission and Detection by Delphinids," In: L. M. Herman, Ed., Cetacean Behavior: Mechanisms and Functions, Wiley-Interscience, New 
York, 1980, pp. 1-52.

[18] S. M. Brownlee, "Correlations between Sounds and Behavior in Wild Hawaiian Spinner Dolphins (Stenella longirostris)," Masters Thesis, University of California Santa Cruz, 1983.

[19] S. M. Dawson, "Clicks and Communication: The Behavioural and Social Contexts of Hector's Dolphin Vocalizations," Ethology, Vol. 88, No. 4, 1991, pp. 265-276.

[20] D. L. Herzing, "Vocalizations and Associated Underwater Behavior of Free-Ranging Atlantic Spotted Dolphins, Stenella Frontalis and Bottlenose Dolphin, Tursiops Truncatus," Aqua Mammal, Vol. 22, 1996, pp. 61-79.

[21] M. H. Rasmussen and L. A. Miller, "Whistles and Clicks from White-Beaked Dolphins, Lagenorhynchus Albirostris, Recorded in Faxaflo'i Bay, Iceland," Aqua Mamm, Vol. 28, 2002, pp. 78-89.

[22] M. O. Lammers, W. W. L. Au and D. L. Herzing, "The Broadband Social Acoustic Signaling Behavior of Spinner and Spotted Dolphins," Journal of the Acoustical Society of America, Vol. 114, No. 3, 2003, pp. 1629-1639. doi:10.1121/1.1596173

[23] M. O. Lammer, M. Schotten and W. W. L. Au, "The Spatial Context of Free-Ranging Hawaiian Spinner Dolphins (Stenella Longirostris) Producing Acoustic Signals," Journal of the Acoustical Society of America, Vol. 119, No. 2, 2006, pp. 1244-1250. doi:10.1121/1.2151804

[24] W. W. L. Au, “The Sonar of Dolphins," Springer-Verlag, New York. 1993.

[25] J. C. Lilly, "Distress Call of the Bottlenose Dolphin: Stimuli and Evoked Behavioral Responses," Science, Vol. 139, 1963, pp. 116-118.

[26] J. J. Dreher and W. E. Evans, "Cetacean Communication," In: W. N. Tavolga, Ed., Marine Bioacousics, Pergammon Press, Oxford, Vol. 1, 1964, pp. 373-399.

[27] M. C. Caldwell, D. K. Caldwell and P. L. Tyack, "Review of the Signature-Whistle Hypothesis for the Atlantic Bottlenose Dolphin," In: S. Leatherwood and R. R. Reeves, Eds., The Bottlenose Dolphin, Academic Press, San Diego, 1990, pp. 199-234.

[28] V. M. Janik, "Whistle Matching in Wild Bottlenose Dolphins Tursiops Truncates," Science, Vol. 289, No. 5483, 2000, pp. 1355-1357.

[29] M. H. Rasmussen, M. Lammers, K. Beedholm and L. A. Miller, "Source Levels and Harmonic Content of Whistles in White-Beaked Dolphins (Langenorhinchus Albirostris)," Journal of the Acoustical Society of America, Vol. 120, No. 1, 2006 pp. 510-517. doi:10.1121/1.2202865

[30] C. D. Hockett, "The Origin of Speech," Scientific American, Vol. 203, 1960, pp. 99-196.

[31] L. M. Herman, D. G. Richards and J. P. Wolz, "Comprehension of Sentences by Bottlenosed Dolphins," Cognition, Vol. 16, No. 2, 1984, pp. 129-219.

[32] L. M. Herman, "Cognition and Language Competencies Of Bottlenosed Dolphins," In: R. J. Schusterman, J.
Thomas and F. G. Wood, Eds., Dolphin Cognition and Behavior: A Comparative Approach, Lawrence Erlbaum Associates, Hillsdale, 1986, pp. 221-251.

[33] L. M. Herman, "What Laboratory Research has Told Us about Dolphin Cognition," International Journal of Comparative Psychology, Vol. 23, 2010, pp. 310-330.

[34] L. M. Herman and W. R. Arbeit, "Frequency Difference Limens in the Bottlenose Dolphin: 1-70 kHz," Journal of Auditory Research, Vol. 12, 1972, pp.109-120.

[35] D. W. Jacobs, “Auditory Frequency Discrimination in the Atlantic Bottlenose Dolphin, Tursiops Truncatus Montague. A Preliminary Report," Journal of the Acoustical Society of America, Vol. 52, No. 2B, 1972, pp. 696-698.

[36] R. K. R. Thompson, and L. M. Herman, "Underwater Frecuency Discrimination in the Bottlenosed Dolphin (1-140khz) and Human (1-8khz)," Journal of the Acoustical Society of America, Vol. 57, 1975, pp. 943-948.

[37] T. H. Bullock, A. D. Grinell, E. Ikezono, K. Kameda, J. Katsuki, M. Nomota, O. Sato, N. Suga and K. Yanagisawa, "Electrophysiological Studies of Central Auditory Mechanisms in Cetaceans," Zeitschrift fiir Vergleichende Phvsiologie, Vol. 59, 1968, pp. 117-156.

[38] C. S. Johnson, “Auditory Masking of One Pure Tone by Another in the Bottlenosed Porpoise," Journal of the Acoustical Society of America, Vol. 49, No. 5, 1970, pp. 1317-1318.

[39] L. M. Herman, P. Morrel-Samuels and A. A. Pack, "Bottlenosed Dolphin and Human Recognition of Veridical and Degraded Video Displays of an Artificial Gestural Language," Journal of Experimental Psychology: General, Vol. 119, 1990, pp. 215-230. doi:10.1037/0096-3445.119.2.215

[40] C. S. Johnson, "Masked Tonal Threshold in the Bottlenose Porpoise," Journal of the Acoustical Society of America, Vol. 44, No. 4, 1968, pp. 965-967. doi:10.1121/1.1911236

[41] C. S. Johnson, M. W. McManus and D. Skaar, "Masked Tonal Hearing Thresholds in the Beluga Whale," Journal of the Acoustical Society of America, Vol. 85, No. 6, 1989, pp. 2651-2654.

[42] W. W. L. Au and P. W. B. Moore, "Critical Ratio and Critical Bandwidth for the Atlantic Bottlenose Dolphin," Journal of the Acoustical Society of America, Vol. 88, No. 3, 1990, pp. 1635-1638. doi:10.1121/1.400323

[43] V. V. Popov, A. Y. Supin and V. O. Klishin, "Frequency Tuning of the Dolphin's Hearing as Revealed by Auditory Brain-Stem Response with Notch-Noise Masking," Journal of the Acoustical Society of America, Vol. 102, No. 6, 1997, pp. 3795-3801.

[44] L. Rendell and H. Whitehead, "Culture in Whales and Dolphins," Behavior and Brain Science, Vol. 24, No. 2, 2001, pp. 309-382.

[45] M. Kruetzen, J. Mann, M. Heithaus, R. Connor, L. Bejder and W. B. Sherwin, "Cultural Transmission of Tool Use In Bottlenose Dolphins," Proceedings of the National Academy of Sciences of the United States of America, Vol. 
105, No. 25, 2005, pp. 8939-8943.

[46] D. F. Bjorklund and J. M. Bering, "Big Brains, Slow Development, and Social Complexity: The Developmental and Evolutionary Origins of Social Cognition," In: M. Bru"ne, H. Ribbert and W. Schiefenho"vel, Eds., The Social Brain: Evolutionary Aspects of Development and Pathology, Wiley, New York, 2003, pp. 133-151.

[47] D. L. Herzing, "Transmission Mechanisms of Social Learning in Dolphins: Underwater Observations of FreeRanging Dolphins in the Bahamas," In: F. Delfour and M. J. Dubois, Eds., Autour de l'ethologie et de la Cognition Animale, Presses Universitaires de Lyon, Lyon, 2005, pp. 185-194.

[48] C. E. Bender, D. L. Herzing and D. F. Bjorklund, "Evidence of Teaching in Atlantic Spotted Dolphins (Stenella frontalis) by Mother Dolphins Foraging in the Presence of Their Calves," Animal Cognition, Vol. 12, No. 1, 2008, pp. 43-53.

[49] D. L. Renaud and A. N. Popper, "Sound Localization by the Bottlenose Porpoise Tursiops Truncates," The Journal of Experimental Biology, Vol. 63, No. 3, 1975, pp. 569-585.

[50] B. K. Branstetter, S. J. Mevissen, L. M. Herman, A. A.
Pack and S. P. Roberts, "Horizontal Angular Discrimination by an Echolocating Bottlenose Dolphin Tursiops Truncates," Bioacoustics, Vol. 14, No. 1, 2003, pp. 1534.

[51] B. K. Branstetter and E. Mercado III,, "Sound Localization by Cetaceans," Journal of Comparative Psychology, Vol. 19, 2006, pp. 26-61.

[52] W. E. Evans, "Vocalizations among Marine Mammals," In: W. N. Tavolga, Ed., Marine Bioacoustics, Pergamon Press, New York, Vol. 2, 1967, pp. 159-186.

[53] M. O. Lammers and W. W. L. Au, "Directionality in the whistles of Hawaiian Spinner Dolphins Stenella Longirostris: A signal Feature to Cue Direction of Movement," Marine Mammal Science, Vol. 19, No. 2, 2003, pp. 249264.

[54] Z. Reznikova, "Analytical Review of Temporal Methodological Approach to the Study of Animal Language Behavior," In: A.D. Koshelev and T. V. Chernigovskaya, Eds., Language and Reasoning. Volume I. Animal Communication and Human Language, Language Origins. Language of Slavonic Cultures, Moscow, 2008, pp. 293337. 